\section{Early diagnosis and successful treatment of small- intestinal carcinoid tumor: useful combination of capsule endoscopy and double-balloon endoscopy}
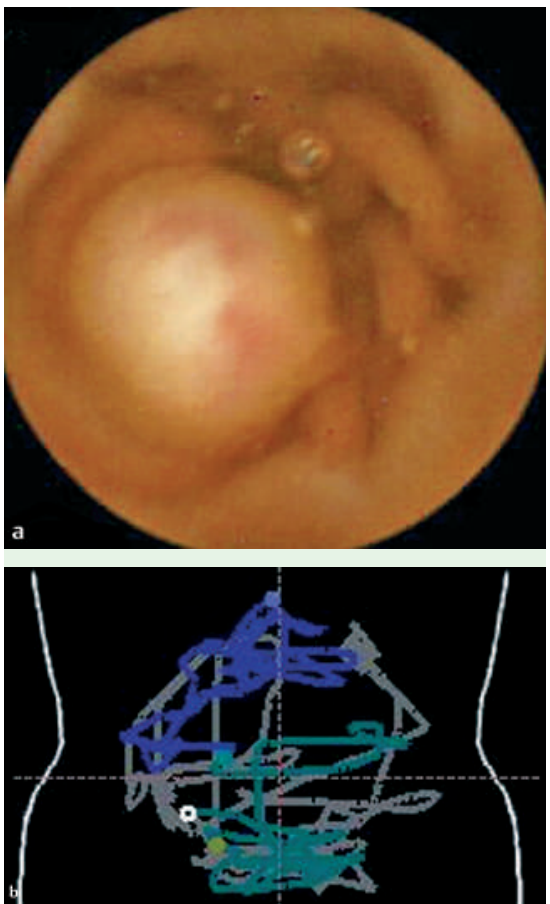

Fig. 1 Capsule endoscopy. a A tumor rising gradually from the mucosa was seen in the proximal ileum. b RAPID software (Given Imaging Ltd., Yoqneam, Israel) showing capsule endoscopy site (white circle).
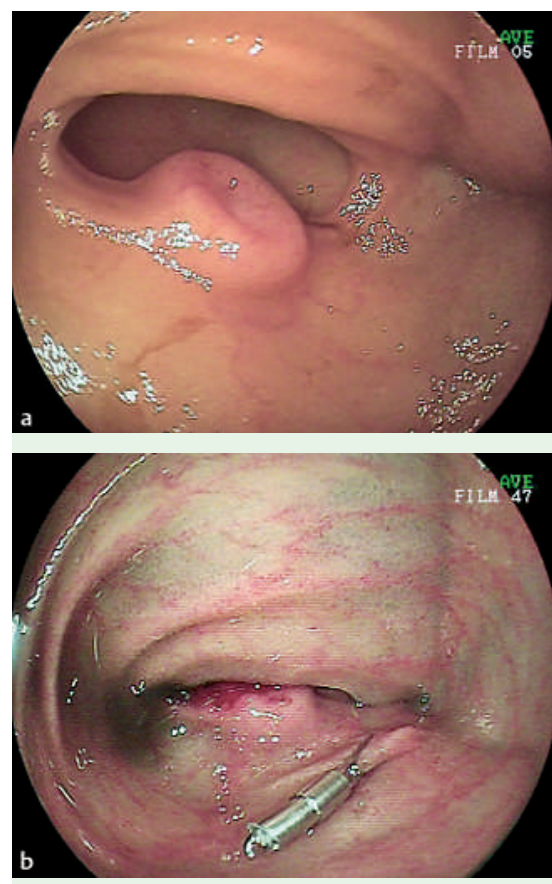

Fig. 2 Double-balloon endoscopy. a A tumor $6 \mathrm{~mm}$ in diameter has a navel-like depression in its center. $\mathbf{b}$ Clips are placed near the tumor after biopsies. tumor and marked it for surgery by placing clips close to it ( $\bullet$ Fig. 2 b).

The patient showed no metastases in a series of imaging examinations and subsequently underwent partial ileectomy. The tumor was completely resected and diagnosed histologically as a carcinoid tumor ( Fig. 3). During the follow-up period, the patient showed no recurrence or metastasis.

The main merit of DBE is that it allows biopsy or transendoscopic procedures [1]. However, DBE requires a very high level of skill and a considerable degree of patient tolerance [2]. It is therefore important to avoid using DBE unnecessarily or inappropriately. In this case, we first screened for the bleeding point by capsule endoscopy and found a tumor in the proximal ileum, for which DBE was subsequently employed transanally and not orally. Thus, screening by capsule endoscopy was useful for deciding the most suitable approach for DBE.

Image resolution in capsule endoscopy has recently improved [3]. Interestingly, in our patient capsule endoscopy detected a small lesion $6 \mathrm{~mm}$ in diameter in the small intestine, suggesting that this procedure is sufficiently useful for screening diseases of the small intestine. On the other hand, DBE has extensive potential for the treatment of hemostasis or dilation of strictures in the small intestine [4]. Thus, capsule endoscopy has certain merits for screening, while DBE seems better suited to final diagnosis or treatment.

Fig. 3 Histological section of the resected carcinoid tumor. The tumor was growing mainly in the submucosa but had also invaded the subserosa.

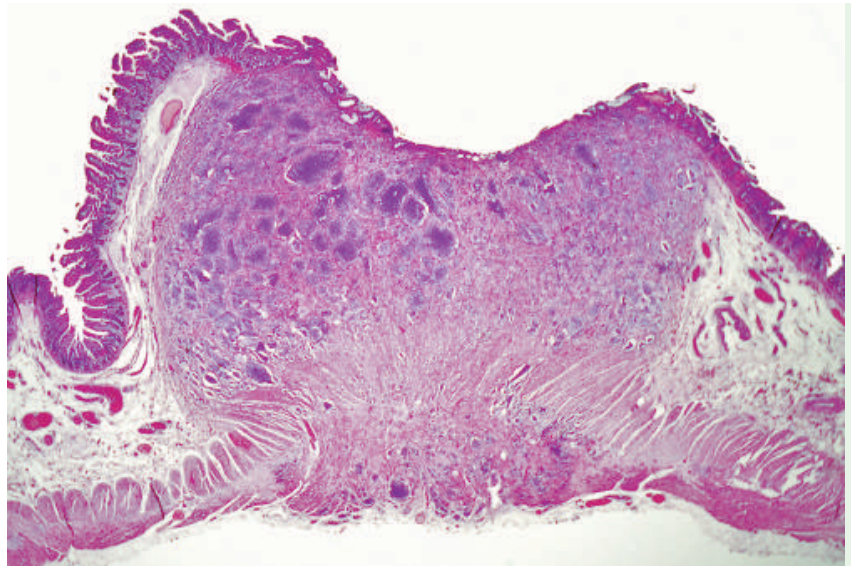

A 38-year-old man with bloody stools underwent esophagogastroduodenoscopy and colonoscopy. However, no bleeding point was detected and therefore, bleeding in the small intestine was considered likely.

We carried out capsule endoscopy and detected a tumor in the proximal ileum
(๑ Fig. 1). Double-balloon endoscopy (DBE) was then carried out transanally and reconfirmed the presence of the tumor in the expected part of the ileum ( $\odot$ Fig. 2 a). On the basis of its appearance, the lesion was suspected to be a submucosal tumor such as a carcinoid. We obtained biopsy samples from the

\section{Acknowledgments $\nabla$}

The authors thank Chiaki Matsuyama, Ayako Shimizu, Takako Otsuki, Midori Katayama, Atsuko Kikuchi, and Sachiko Miyahara (Department of Surgical and Molecular Pathology, Dokkyo Medical University School of Medicine, Tochigi, Japan) for their excellent technical and secretarial assistance.

Endoscopy_UCTN_Code_CCL_1AC_2AC 
H. Yamagishi ${ }^{1,2}$, H. Fukui ${ }^{1}$, K. Shirakawa $^{2,3}$, T. Oinuma ${ }^{2}$, H. Hiraishi ${ }^{2}$, A. Terano ${ }^{2}$, T. Fujimori ${ }^{1}$, T. Nakamura ${ }^{3}$ 1 Department of Surgical and Molecular Pathology, Dokkyo Medical University School of Medicine, Tochigi, Japan

2 Department of Gastroenterology, Dokkyo Medical University School of Medicine, Tochigi, Japan

3 Department of Endoscopy, Dokkyo Medical University School of Medicine, Tochigi, Japan

\section{References}

1 Hadithi M, Heine GD, Jacobs MA et al. A prospective study comparing video capsule endoscopy with double-balloon enteroscopy in patients with obscure gastrointestinal bleeding. Am J Gastroenterol 2006; 101 : $52-57$

2 Mehdizadeh S, Ross A, Gerson L et al. What is the learning curve associated with doubleballoon enteroscopy? Technical details and early experience in 6 US tertiary care centers. Gastrointest Endosc 2006; 64: 740 750

3 Technology Assessment Committee, American Society for Gastrointestinal Endoscopy. ASGE Technology Status Evaluation Report: wireless capsule endoscopy. Gastrointest Endosc 2006; 63: 539-545

4 Yamamoto H, Kita H, Sunada K et al. Clinical outcome of double balloon endoscopy for the diagnosis and treatment of small-intestinal diseases. Clin Gastroenterol Hepatol 2004; 2: 1010-1016
Bibliography

DOI $10.1055 / \mathrm{s}-2007-966620$

Endoscopy 2007; 39: E243-E244

(c) Georg Thieme Verlag KG Stuttgart · New York . ISSN 0013-726X

\section{Corresponding author}

\section{T. Nakamura, MD PhD FACG}

Department of Endoscopy

Dokkyo Medical University School of Medicine 880 Kitakobayashi

Mibu Shimotsuga

Tochigi 321-0293

Japan

Fax: +81-282-86-7761

nakamurt@dokkyomed.ac.jp 